$$
\begin{aligned}
\int_{a}^{b} x^{n} \psi(x) d x= & a_{0} \int_{a}^{b} \psi(x) d x+a_{1} \int_{a}^{b} \psi(x) \cos x d x \\
& +b_{1} \int_{a}^{b} \psi(x) \sin x d x+\cdots=0 \quad(n=1,2, \cdots) .
\end{aligned}
$$

Hence $\psi(x)$ satisfies the conditions of Theorem I, and therefore it is zero at every point at which it is continuous.

The method of proof used in Theorem II may be applied in the case of developments in terms of any other normal functions, such as Bessel functions, Legendre's polynomials, etc., whenever we know that $x^{n}$ can be developed in a convergent series of such functions which, when differentiated term by term, will yield a uniformly convergent series that represents the derivative of $x^{n}$.* The method will enable us to show in such cases that if the coefficients of the development corresponding to any function which we know to be finite, save for a finite number of points, and integrable, are all zero, the function is zero at every point at which it is continuous.

\title{
NOTE ON THE SECOND VARIATION IN AN ISOPERIMETRIC PROBLEM.
}

BY DR. ELIJAH SWIFT.

(Read before the American Mathematical Society, April 25, 1908.)

SuPPOSE we have before us the simplest type of isoperimetric problem, namely to determine $x$ and $y$ as functions of a parameter $t$, so that the definite integral

$$
J=\int_{t_{0}}^{t_{1}} F\left(x, y, x^{\prime}, y^{\prime}\right) d t
$$

shall be a minimum, while another definite integral

$$
K=\int_{t_{0}}^{t_{1}} G\left(x, y, x^{\prime}, y^{\prime}\right) d t
$$

* The existence of such developments can be proved for some of these cases by means of some theorems discussed by Stekloff. Cf. Mémoires de l'Académie de St. Pétersbourg, ser. 8, vol. 15 (1904). 
takes a given value $l$, the functions $x(t)$ and $y(t)$ being subject to the condition that they take given values $x_{0}, y_{0}$ and $x_{1}, y_{1}$ for $t=t_{0}$ and $t=t_{1}$ respectively.

The object of this note is to point out a way of obtaining the second variation of $J$ that seems to me clearer than that found in most text-books, ${ }^{*}$ and which supplies the reasons for the usual derivation.

If we have a solution of the problem, $x=x(t), y=y(t)$, we vary the integral $J$ by replacing $x$ and $y$ by $x+\xi$ and $y+\eta$ respectively, where for all variations that leave $K$ unchanged $\xi$ and $\eta$ may be expressed as power series in a quantity $\epsilon_{1}$, if for convenience sake we assume that $K$ and $F$ are analytic, and taking $\epsilon_{1}$ as principal infinitesimal, are in general of the first order. $\dagger$ We thus obtain a new integral $J+\Delta J$. The first variation of $J$ consists of all terms of $\Delta J$ which are of the first order ; the second variation, of all those of the second order, and so on.

To obtain the second variation Bolza proceeds as follows: Since for all admissible variations $K$ must remain equal to $l$, we must have $\Delta K=0 . \Delta J$ is therefore unchanged if we add any multiple of $\Delta K$ to it, in particular $\Delta J=\Delta J+\lambda_{0} \Delta K$, where $\lambda_{0}$ is the isoperimetric constant. $\S$ Developing this expression and taking the terms of the second degree in $\xi, \eta$ and their derivatives we have the usual expression for the second variation $\Delta^{2} J$.

We naturally ask: Why add $\Delta K$ at all to $\Delta J$ ? And why add exactly $\lambda_{0} \Delta K$ ? Moreover if $\Delta K=0$, how does it happen that adding it to $\Delta J$ makes any difference at all in the second variation? In considering a special isoperimetric problem $\| \mathrm{I}$ was led to attempt to answer these questions.

The total variation of $J$ is

$$
\begin{aligned}
\Delta J=\int_{t_{0}}^{t_{1}}\left[\left(\frac{\partial F}{\partial x} \xi+\right.\right. & \left.\frac{\partial F}{\partial y} \eta+\frac{\partial F}{\partial x^{\prime}} \xi^{\prime}+\frac{\partial F}{\partial y^{\prime}} \eta^{\prime}\right) \\
& \left.+\frac{1}{2}\left(\frac{\partial^{2} F}{\partial x^{2}} \xi^{2}+\cdots+\frac{\partial^{2} F}{\partial y^{\prime 2}} \eta^{\prime 2}\right)+\cdots\right] d t .
\end{aligned}
$$

*E. g., Bolza, "Lectures on the Calculus of Variations," p. 216.

$\dagger$ Bolza, pp. $206 \mathrm{ff}$.

$\ddagger$ Bolza, loc. cit.

\& Bolza, pp. 208, 209.

|| Dissertation, Göttingen, 1907, pp. 16 ff. 
To find the second variation, we must take all terms that are of the second order. But these come, not merely from the terms of the second degree in $\xi, \eta$ and their derivatives, but also from those of the first, since $\xi$ and $\eta$ are power series in $\epsilon_{1}$ and involve other powers of $\epsilon_{1}$ besides the first. We must take then all terms of the second order in $\Delta J$. Then we have $\Delta^{2} J=$ all terms of the second order in

$$
\int_{t_{0}}^{t_{1}}\left[\left(\frac{\partial F}{\partial x} \xi+\cdots+\frac{\partial F}{\partial y^{\prime}} \eta^{\prime}\right)+\frac{1}{2}\left(\frac{\partial^{2} F}{\partial x^{2}} \xi^{2}+\cdots+\frac{\partial^{2} F^{\prime}}{\partial y^{\prime 2}} \eta^{\prime 2}\right)\right] d t .
$$

The question comes up-How pick out the terms of the second order in the first term in $\Delta J$ and so obtain an explicit expression for the second variation?

We can do this by using the fact that since $\Delta K=0$, all the terms of the second order in $\Delta K$ must vanish, and moreover the first variation of $\Delta J$ is zero.* The two corresponding equations are $\Delta^{2} K=$ all terms of the second order in

$\int_{t_{0}}^{t_{1}}\left[\left(\frac{\partial G}{\partial x} \xi+\cdots+\frac{\partial G}{\partial y^{\prime}} \eta^{\prime}\right)+\frac{1}{2}\left(\frac{\partial^{2} G}{\partial x^{2}} \xi^{2}+\cdots+\frac{\partial^{2} G}{\partial y^{\prime 2}}{\eta^{\prime 2}}^{2}\right)\right] d t=0$

and

$$
\int_{t_{0}}^{t_{1}}\left[\left(\frac{\partial F}{\partial x} \xi+\cdots+\frac{\partial F}{\partial y^{\prime}} \eta^{\prime}\right)+\lambda_{0}\left(\frac{\partial G}{\partial x} \xi+\cdots+\frac{\partial G}{\partial y^{\prime}} \eta^{\prime}\right)\right] d t=0 .
$$

It is at once evident that we can eliminate the terms of the first degree in $\Delta J$ by adding to it $\lambda_{0} \Delta K$, and so obtain an explicit expression for the second variation. If we call $F+\lambda_{0} G=H$, we have $\Delta^{2} J=\Delta^{2} J+\lambda_{0} \Delta^{2} K=$ all terms of the second order in

$$
\frac{1}{2} \int_{t_{0}}^{t_{1}}\left(\frac{\partial^{2} H}{\partial x^{2}} \xi^{2}+\cdots+\frac{\partial^{2} H}{\partial y^{\prime 2}} \eta^{\prime 2}\right) d t
$$

the form in which the second variation is usually written.

The advantage of adding $\lambda_{0} \Delta K$ to $\Delta J$ is then that by so doing we obtain the terms of the second order in the isoperimetric problem explicitly from $H$ in the same way as in the ordinary problem from $F$.

Princeton, N. J., February, 1908.

* Bolza, p. 209. 